\title{
Characterization of allergenic epitopes of Ory s1 protein from Oryza sativa and its homologs
}

\author{
Ruchi Sharma $^{1, *}$, Ashok Kumar Singh ${ }^{1}$, Vetrivel Umashankar ${ }^{2}$ \\ ${ }^{1}$ Department of Botany, Udaya Pratap College, Varanasi, Uttar Pradesh, India; ${ }^{2}$ Department of Bioinformatics, School of Biosciences, SRM \\ University, Ramapuram, Chennai, Tamil Nadu, India; Ruchi Sharma - Email: ruchivns@rediffmail.com; * Corresponding author
}

Received March 16, 2009; Revised May 04, 2009; Accepted June 17, 2009; Published August 18, 2009

\begin{abstract}
:
Vaccination is the most effective technique suggested now days for allergy treatment. Recombinant-based approaches are mostly focused on genetic modification of allergens to produce molecules with reduced allergenic activity and conserved antigenicity. The molecules developed for vaccination in allergy possess significantly reduced allergenicity in terms of IgE binding, and therefore will not lead to anaphylactic reactions upon injection. This approach is probably feasible with every peptide allergen with known amino acid sequence. In this study an in silico approach was used to investigate allergenic protein sequences. Motif analysis of these sequences reveals the allergenic epitopes in the amino acid sequences. Physicochemical analysis of protein sequences shows that the homolog allergens of Ory s1 are highly correlated with the aromaticity, GRAVY and cysteine content. Moreover, phylogenetic analysis of Ory s1 with other sequences reveals that Oryza sativa japonica and Zea mays are close homologs, whilst Lolium perenne and Dactylis glomerata are found to be remote homologs. The multiple sequence alignment reveals of Ory s1 with all its homologs in this study reveals the high conservation of residues in DPBB_1 domain (amino acid residue positions 86- 164) and was found distinctly in all the sequences. These findings support the proposal that allergenic epitopes encompass conserved residues. The consensus allergenic was found to be mainly composed of hydrophobic residues. The functional sites of allergenic proteins reported in this study shall be attenuated to develop hypoallergenic vaccine. The sequence comparison strategy adopted in this study would pave way effective evolutionary analysis of these allergens.
\end{abstract}

Keywords: Ory s1, sequence analysis, physicochemical analysis, allergenic epitopes, phylogenetic analysis.

\section{Background:}

The Ory s1 protein from Oryza sativa has been studied extensively to gain a better understanding of its remarkable allergenicity [1]. Pollen allergens of Oryza sativa is recognized by the International Union of Immunological Societies (IUIS) official list of allergens which include Ory s1, Ory s7, and Ory s12. Much information has been published focusing on the physicochemical and epitope analysis of the allergenic pollen proteins [2, 3, 4]. Majority of the world's population depend on rice, wheat, maize for daily sustenance. These provide important models for evolutionary studies of the grasses since various aspects of their biology have been well documented [5]. The present investigation focuses on sequence and epitope analysis of Ory s1 and its homologs. Chemical modification of allergen vaccines to reduce IgE binding improves safety while maintaining clinical efficacy. Analysis of molecular size and allergen content may be useful techniques for characterization and standardization of allergoid products [6]. Identification of potentially allergenic proteins is needed for the safety assessment of genetically modified foods, certain pharmaceuticals and various other products on the consumer market. Features that differentiate allergens from non-allergens are difficult to find by manual inspection of amino acid sequences. Current methods in bioinformatics allergology exploit common features among allergens for the detection of amino acid sequences of potentially allergenic proteins. Features for identification still unexplored include the motifs occurring commonly in allergens, but rarely in ordinary proteins [7]. In addition to laboratory experimentation and clinical testing, current procedures for allergenicity assessment involve an introductory comparison of the novel protein's amino acid sequence with those of known allergens [8]. Several regions of the amino acid sequence of the homologs are evolutionarily highly conserved. Highly conserved primary sequences of allergenic homologs have been used in an attempt to establish evolutionary relationships. The studies of different allergen protein sequences suggest that allergens tend to share certain sequence similarities. Thus, the potential allergenicity of query proteins can be predicted by examining their sequence similarities with known allergens [9]. The application of computational techniques in biological discovery was possible due to the availability of extensive sequence data. The most widely-used and

ISSN 0973-2063 (online) 0973-8894 (print)

Bioinformation 4(1): 12-18 (2009) conceptually easiest to understand of these techniques is database homology searching, where sequence similarity can be used to assign target for hypoallergenic vaccine production. A recent paper showed the efficacy of recombinant birch pollen vaccine for the treatment of birch-allergic rhinoconjunctivitis [10]. The similarities found between the homologous sequences argue that there should be similarities in their three-dimensional structures, strengthening the hypothesis that proteins with similar sequences perform a similar function. The main objective of this study is to analyze the comparative abundance and distribution of allergenic epitopes in the sequences and to help in identifying target amino acid positions in the course of vaccine development.

\section{Methodology:}

\section{Sequence retrieval:}

All databases and software used in these studies are publicly available on the world-wide web. The primary sequence of Ory s1 from Oryza sativa was acquired from the NCBI's GenPept, a publicly available database [11]. BLAST (psi blast) search, using the non redundant database, was performed that resulted homologous sequences. Twenty homologs from distant organisms were selected and the sequences were acquired from GenPept. This provided data required to predict the primary structure (sequence) and to perform the allergenicity assessment study.

Physiochemical analysis:

Physiochemical analysis of the sequences namely molecular weight, theoretical pI, amino acid composition, instability index, aliphatic index and grand average of hydropathicity (GRAVY) were done using PROTPARAM tool [12].

\section{Phylogenetic analysis:}

Clustal W analysis software available online from Moscow State University's A. N. Belozersky Institute of Physio-Chemical Biology was used to compare sequence alignment of allergenic homologs with the default settings [13]. Here the dendrogram is calculated in 2 stages: first all pairs of sequence are compared using of Wilbur and Lipman method [14] and then the similarity scores resulted are used to 
construct the dendrogram using the UPGMA cluster analysis method of Sneath and Sokal [15]. Tree construction was done by using Phylodraw [16].

\section{Allergenic domain detection:}

Allergenic domains of the sequences were extracted using ProScan [17]. Motif search in the multiple sequence alignment was carried out using Multiple Em for Motif Elicitation (MEME tool) technique [18]. Antigenic sites on proteins was discovered, using EMBOSS antigenic program [19], a semi-empirical method which makes use of physicochemical properties of amino acid residues and their frequencies of occurrence in experimentally known segmental epitopes [20].

\section{Discussion:}

Sequence from NCBI server was retrieved for Oryza sativa allergenic protein Ory S1 A86533.1. BLAST search (Psi BLAST) obtained homologous Sequences, and 20 significant homologous sequences were short listed from diverse species varying from grasses to higher plants for comparative study: AAA86533.1, NP_001048686.1, CAA81613.1, CAA10520.1, CAA10140.1, CAB63699.1, AAP96760.1, AAS48882.1, ABF81662.1, CAC40805.1, ABB83474.1, AAZ08315.1, NP_190182.2, ABK93417.1, AAV85475.1, ACB45301.1, ABJ90221.1, AAT11859.2, BAC67192.1, BAC66787.1. Amino acid residues Ala (7.6-11.9), Cys (2.70-3.80), His (1.0-2.2), Leu (4.6-6.7), Trp (2.2-3.5), Tyr (2.3-4.5), Val (6.2-9.8) are found quite equally distributed among all the sequence than other amino acids (Table 1), while Cys and Val residues are predominant found in allergenic epitopes (Table 4). Table 4 shows most of the hydrophobic residues in allergenic site. Table 2 provides details of the physiochemical analysis which shows all the sequences as stable (instability index ranging from 17.35 to 44.02 ) with a theoretical pI ranging from 5.32 to 9.58 . Though GRAVY was found negative (-.003 to -0.643) for complete sequences the distribution of sequences in alleregnic motif was predominantly hydrophobic (Table 1). The information content diagram provides an idea of the positions in the motif that are most highly conserved. It is very interesting to note the simplified position specific matrix result where the four conserved cystiene residues was found in all the sequences at the same position (Table 3). Interestingly the phylogenetic tree of Ory s1 homologs included several apparently eukaryotic orthologs. Therefore it seems most likely that the progenitors of each of these orthologous sets might also cause allergy. The phylogenetic analysis has resulted that pollen allergen sequence from Oryza sativa japonica (NP_001048686.1) was most closely related sequence and EXPB10 sequence from Zea mays (ABF81662.1) as next closely related sequence with the query sequence Ory s1 from Oryza sativa indica (AAA86533.1). Beta expansin B2 from Festuca pratensis (CAC40805.1) and Beta-expansin EXPB4 from Hordeum vulgare (ACB45301.1) were next most closely related sequences found. Expansin 1 from Mangifera indica (AAT11859.2) and sequence from Eucalyptus globulus (AAZ08315.1) were found less related. Pollen allergen from Lolium perenne (CAB63699.1) and Group 1 allergen Dac g 1.02 precursors from Dactylis glomerata (AAP96760.1) were found as distantely related. It shows that the relation ship of the sequences in phylogenetic analysis (Figure 1).

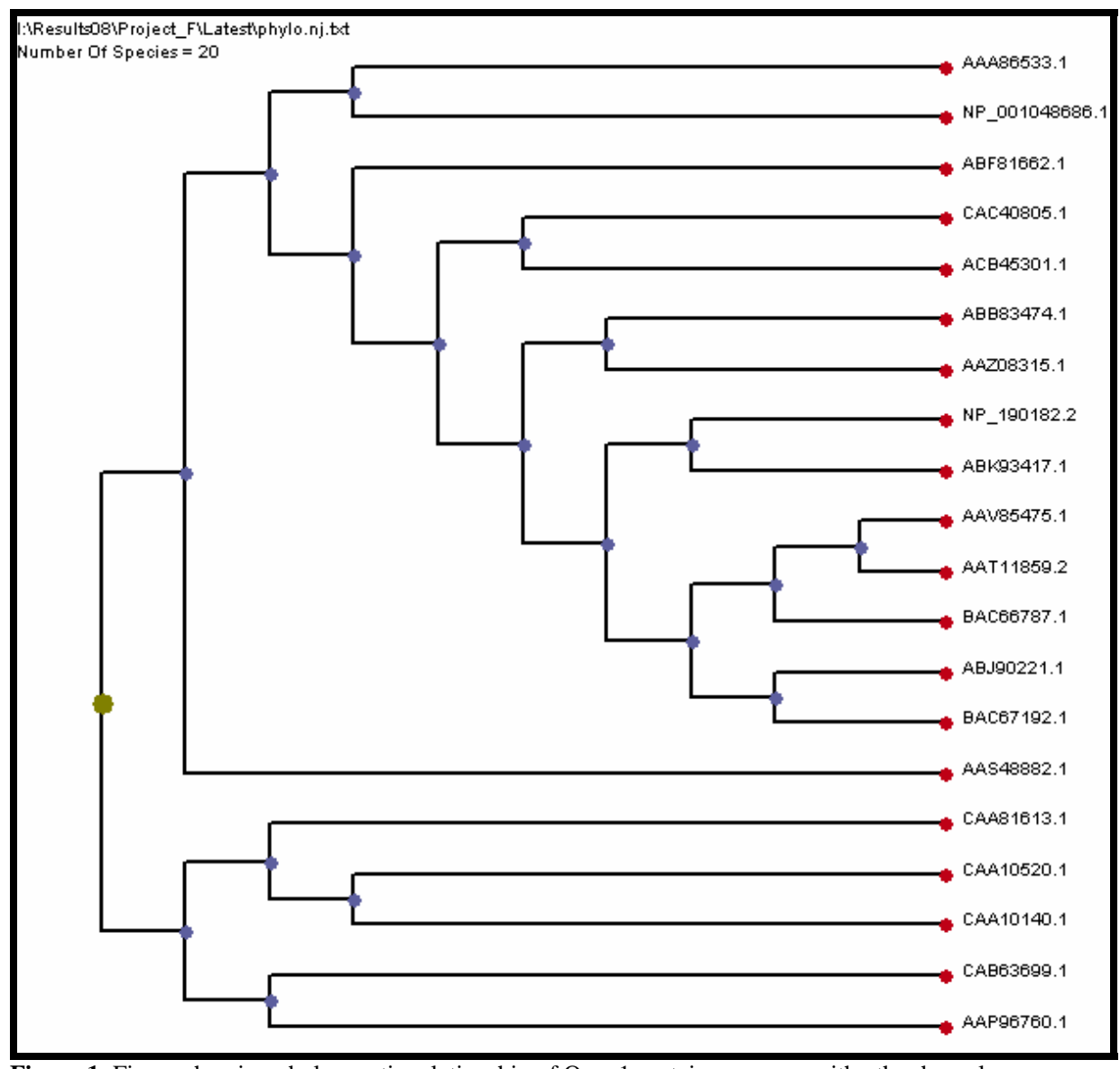

Figure 1: Figure showing phylogenetic relationship of Ory s1 protein sequence with other homologs. 


\section{Bioinformation}

\section{Conclusion:}

Consensus epitope identification using the accessible allergenic region has geared up the pace. If bioinformatics approaches are standardized and optimized, it can be used for the swift identification of potential antigenic regions to target allergenic proteins in course of development of hypoallergenic vaccines. Allergenic epitopes in this study show abundance of hydrophobic residues in the motif. The role of highly conserved cysteines residues at four positions shall also play a major role in determining the allergenicity. Cystiene residues were found highly conserved for the motif 1 with a width of 29 and 20 sites. It was found in all the sequences at $9,21,24,29$ positions as shown in the information content diagram [21]. This is in order with the results documented in table 3 . The function of disulfide bonds formed between cysteines in IgE binding has been investigated in studies with several other allergens this falls in line with the previously documented studies [22, 23]. Hence, the procured consensus region shall be utilized for effective vaccine design against food allergens.

\section{References:}

[1] D Scott et al., Molecular Plant 1:751 (2008) [PMID: 2660330]

[2] F Takaiwa et al, Immunol. Allergy Clin. North Am. 27:129 (2007) [ PMID: 17276883 ]

[3] JS Ye et al, DNA Research 12:167 (2005) [PMID: 16303748]

[4] MM Sen et al., J. Environ. Monit. 5:959 (2003) [PMID: 14710939]

[5] P Rajendrakumar et al., In Silico Biology 8: 9 (2008) [PMID: 18928198]
[6] J Carnes et al., Clin. Exp. Allergy 39:426 (2009) [PMID:19134021]

[7] K Bjorklund et al., Bioinformatics 21:39 (2005) [PMID: 15319257]

[8] W Kong et al., In Silico Biology 7:77 (2006) [PMID: 17688432]

[9] GS Ladics, MK Selgrade, Regul Toxicol Pharmacol. 54:S2. (2009) [PMID: 19028539]

[10] G Pauli et al, J. Allergy Clin. Immunol. 122:951 (2008) [PMID: 19000581]

[11] http://blast.ncbi.nlm.nih.gov

[12] http://www.expasy.ch/tools/protparam.html

[13] http://www.genebee.msu.su/clustal/

[14] WJ Wilbur, DJ Lipman, Acad. Sci. Vold., 80:726 (1983)

[15] PHA Sneath, RR Sokal, Numerical Taxonomy: Freeman, San Francisco (1973)

[16] http://pearl.cs.pusan.ac.kr/phylodraw

[17] http://npsa-pbil.ibcp.fr/

[18] http://meme.sdsc.edu/meme/meme-intro.html

[19] http://inn.weizmann.ac.il/cgibin/EMBOSS/

[20] AS Kolaskar, PC Tongaonkar, FEBS Lett. 276:172 (1990) [PMID: 1702393]

[21] R Sharma, AK Singh, Am. J. Infectious Diseases 5:149 (2009)

[22] M Lombardero et al., J. Immunol. 144:1353 (1990) [PMID: 1689351]

[23] S Olsson et al., Mol. Immunol. 35:1017 (1998) [PMID: 10068036]

Edited by $P$. Kangueane

Citation: Sharma et al, Bioinformation 4(1): 12-18 (2009) License statement: This is an open-access article, which permits unrestricted use, distribution, and reproduction in any medium, for noncommercial purposes, provided the original author and source are credited. 
Supplementary material

Table 1: Table showing amino acid composition table (\%) for the residues found most conserved in the homologs

\begin{tabular}{lllllllll}
\hline S. No & Organism Name & Ala (A) & Cys (C) & His(H) & Leu (L) & Val(V) & Trp(W) & Tyr (Y) \\
\hline 1 & AAA86533.1 & 9.90 & 3.00 & 1.50 & 5.70 & 7.60 & 1.50 & 2.30 \\
2 & NP_001048686.1 & 10.60 & 3.40 & 1.10 & 5.30 & 6.80 & 2.30 & 3.00 \\
3 & CAA81613.1 & 8.70 & 2.70 & 1.50 & 4.60 & 8.00 & 2.30 & 3.00 \\
4 & CAA10520.1 & 8.40 & 2.70 & 1.50 & 5.70 & 7.20 & 2.30 & 3.40 \\
5 & CAA10140.1 & 8.00 & 2.70 & 1.50 & 6.10 & 8.00 & 2.30 & 3.40 \\
6 & CAB63699.1 & 8.00 & 2.70 & 1.50 & 4.90 & 7.20 & 2.30 & 3.40 \\
7 & AAP96760.1 & 7.60 & 2.70 & 1.90 & 5.30 & 7.60 & 2.30 & 3.40 \\
8 & AAS48882.1 & 7.80 & 3.00 & 2.20 & 4.40 & 7.80 & 2.20 & 3.30 \\
9 & ABF81662.1 & 7.80 & 3.30 & 1.10 & 5.20 & 8.60 & 2.60 & 4.50 \\
10 & CAC40805.1 & 10.40 & 6.30 & 2.20 & 5.90 & 5.90 & 2.20 & 4.10 \\
11 & ABB83474.1 & 8.00 & 3.60 & 1.10 & 6.50 & 8.00 & 2.20 & 3.30 \\
12 & AAZ08315.1 & 11.90 & 3.80 & 1.00 & 6.70 & 8.60 & 1.90 & 2.90 \\
13 & NP_190182.2 & 8.80 & 3.30 & 1.40 & 5.60 & 9.80 & 2.30 & 4.20 \\
14 & ABK93417.1 & 9.30 & 4.20 & 1.20 & 5.80 & 7.30 & 2.30 & 3.50 \\
15 & AAV85475.1 & 8.50 & 3.10 & 1.60 & 6.60 & 7.00 & 3.10 & 3.50 \\
16 & ACB45301.1 & 10.30 & 3.30 & 2.20 & 6.20 & 6.20 & 2.60 & 4.80 \\
17 & ABJ90221.1 & 10.30 & 3.20 & 2.40 & 5.50 & 6.70 & 2.80 & 4.70 \\
18 & AAT11859.2 & 8.80 & 3.80 & 1.20 & 5.40 & 6.90 & 3.50 & 3.50 \\
19 & BAC67192.1 & 9.90 & 3.20 & 2.40 & 5.90 & 7.10 & 2.80 & 4.70 \\
20 & BAC66787.1 & 9.20 & 3.50 & 1.20 & 5.40 & 8.10 & 3.50 & 3.10 \\
\hline
\end{tabular}

Table 2: Table showing physiochemical properties of Ory s1 and its homologous sequences

\begin{tabular}{llllllllll}
\hline SN & Accession No. & MW & NCR & PCR & TP & II & AI & G \\
\hline 1 & AAA86533.1 & 28497.70 & 34 & 38 & 8.53 & 44.02 & 73.46 & -0.41 \\
2 & NP_001048686.1 & 28351.40 & 36 & 35 & 6.34 & 31.01 & 73.22 & -0.33 \\
3 & CAA81613.1 & 28203.10 & 33 & 34 & 7.53 & 25.33 & 68.97 & -0.32 \\
4 & CAA10520.1 & 28246.00 & 35 & 34 & 6.46 & 25.62 & 66.39 & -0.393 \\
5 & CAA10140.1 & 28347.20 & 36 & 35 & 6.46 & 22.07 & 69.70 & -0.388 \\
6 & CAB63699.1 & 28360.90 & 37 & 33 & 5.69 & 25.47 & 64.52 & -0.454 \\
7 & AAP96760.1 & 28468.10 & 36 & 33 & 6.01 & 25.04 & 65.00 & -0.446 \\
8 & AAS48882.1 & 29026.90 & 32 & 34 & 8.02 & 28.96 & 65.00 & -0.423 \\
9 & ABF81662.1 & 29109.40 & 30 & 33 & 8.25 & 17.35 & 73.20 & -0.249 \\
10 & CAC40805.1 & 29307.00 & 22 & 23 & 7.60 & 36.29 & 69.70 & -0.219 \\
11 & ABB83474.1 & 28983.50 & 19 & 19 & 6.79 & 42.20 & 68.07 & -0.106 \\
12 & AAZ08315.1 & 21755.40 & 17 & 14 & 5.32 & 34.43 & 72.05 & -0.042 \\
13 & NP_190182.2 & 23391.70 & 16 & 24 & 9.14 & 31.65 & 80.70 & -0.148 \\
14 & ABK93417.1 & 28470.30 & 25 & 28 & 8.25 & 38.92 & 74.21 & -0.147 \\
16 & AAV85475.1 & 27917.60 & 11 & 20 & 9.34 & 35.85 & 71.09 & -0.056 \\
& ACB45301.1 & 30067.30 & 17 & 31 & 9.49 & 34.23 & 64.03 & -0.358
\end{tabular}




$\begin{array}{lllllllll}17 & \text { ABJ90221.1 } & 26800.00 & 6 & 17 & 9.48 & 35.50 & 60.59 & -0.163 \\ 18 & \text { AAT11859.2 } & 28333.40 & 11 & 23 & 9.48 & 40.16 & 67.92 & 0.003 \\ 19 & \text { BAC67192.1 } & 26755.00 & 6 & 16 & 9.38 & 35.28 & 62.89 & -0.125 \\ 20 & \text { BAC66787.1 } & 28019.80 & 10 & 22 & 9.58 & 33.98 & 68.65 & -0.008\end{array}$

SN = S. No.; MW = molecular weight; NCR = negatively charged residues; PCR = positively charged residues; TP = Theoretical pI; II = Instability index; AI = Aliphatic index; G = Gravity

Table 3: Simplified position-specific probability matrix for motif 1

Simplified position-specific probability matrix

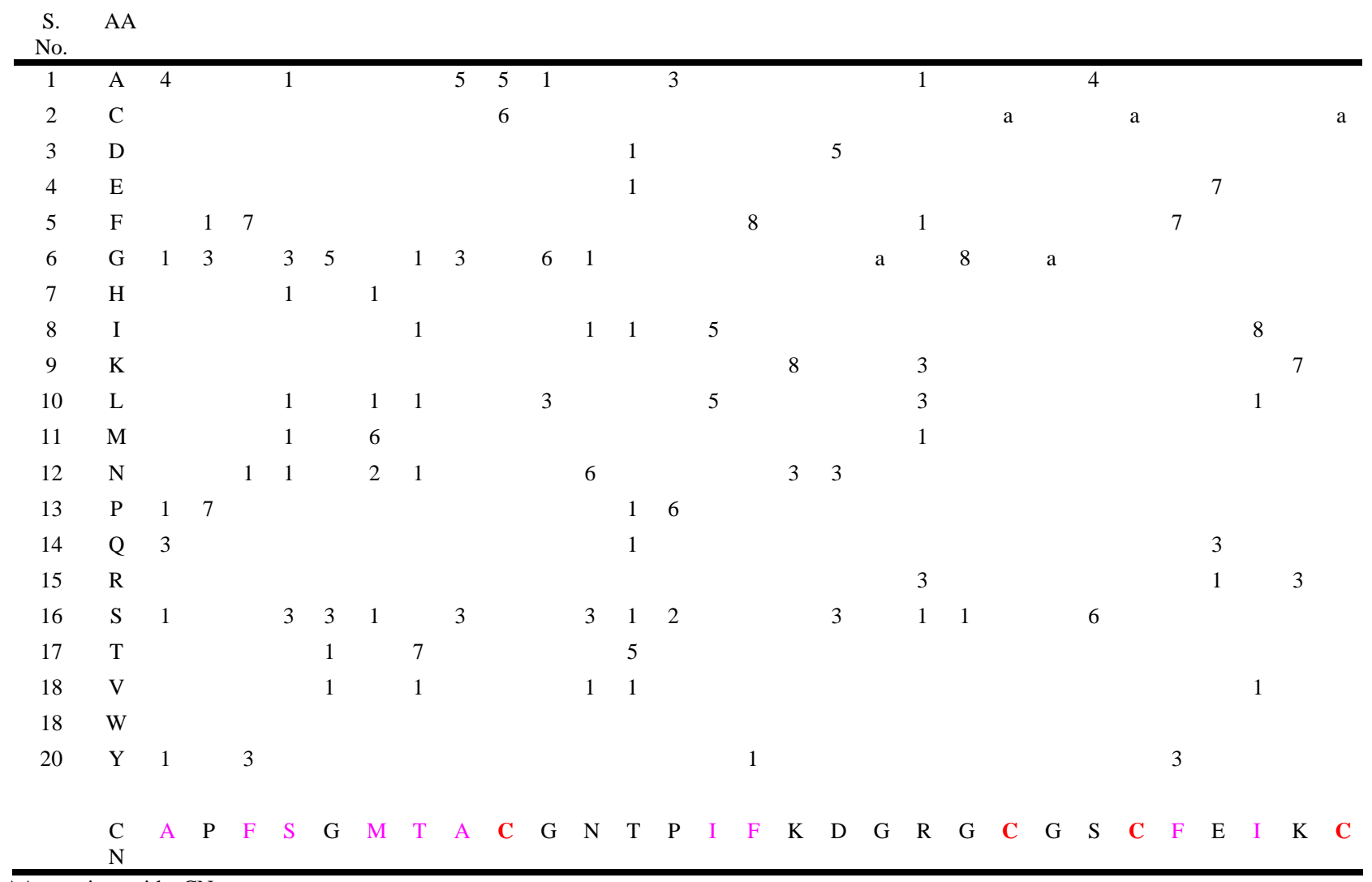

AA = amino-acids; $\mathrm{CN}=$ consensus

Table 4: Table showing five major allergenic epitopes in protein homologs of Ory s 1 using EMBOSS antigenic program

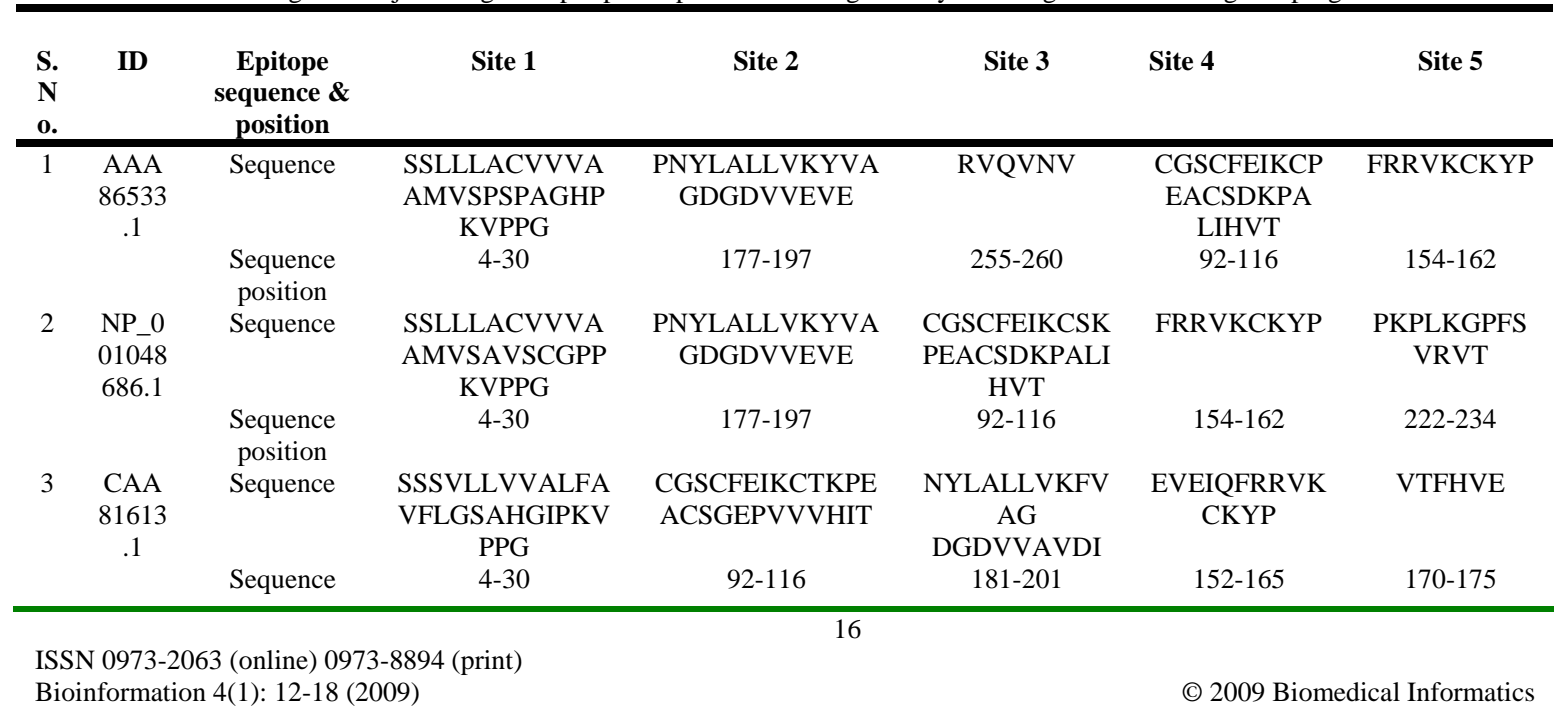




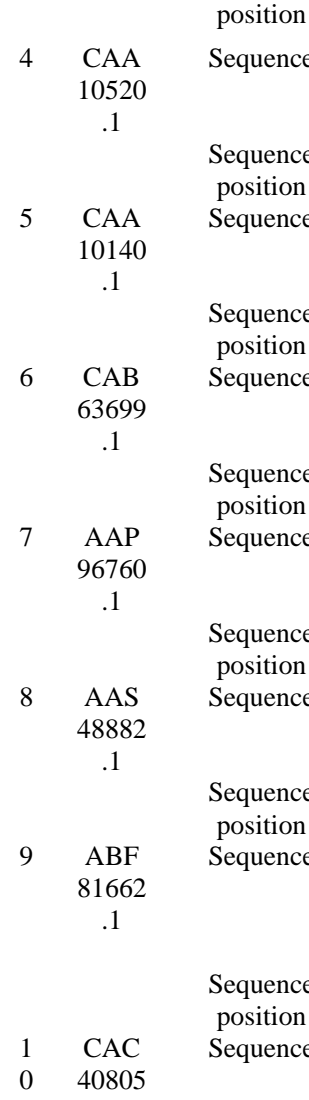

.1

Sequence

position

1 ABB Sequence

$1 \quad 83474$

1

$1 \quad$ AAZ

208315

.1

$\begin{array}{ccc}\text { NP_1 } & \begin{array}{c}\text { Sequence } \\ \text { position } \\ \text { Sequence }\end{array} \\ 3 & \text { N0 } & \\ & 182.2 & \text { Sequence } \\ & & \begin{array}{c}\text { position } \\ \text { Sequence }\end{array} \\ 1 & \text { ABK } & \text { Sequence } \\ 4 & 93417 & \\ & .1 & \\ & & \text { Sesition } \\ & & \text { Sequence } \\ 1 & \text { AAV } & \\ 5 & 85475 & \\ & .1 & \text { Sequence } \\ & & \text { position }\end{array}$

Sequence

position

Sequence

Sequen

(a)

SSSVLLVVALFA
VFLGTAHGIAK
VPPG

4-30

SSLVLLVVALF

AVFLGTAHGIA

KVPPG

4-30

SSSVLLVVALFA

VFLGSAHGIAK

VPPG

4->30

SSSVLLVVALFA

VFLGSAHGIPKV

PPG

5-31

SSSVLLVAAVL AAVVCG

AHGIAKVPPG

5-31

VSIMWSLVQVQ

VLVAVAL

SFLVGGAWCGP PKVPPG

4-38

HSGIIDIQFRRVP

CNFPGLKINFHV

VDGSNAVYLAV LIE

152-90

GCGACYQVKCT

$29-43$
GCGACFQVRCK
NPKLCNSKGTIV
MVT

FICFLFLAISYAT

ACDRCVHQSKV

AYFSRDSALSSG ACGYG

4-44

ATISFISLVLLLS

LVEARIPGVYT

$\mathrm{G}$

5-29
NYLALLVKYV

181-190

NYLALLVKYV

181-190

PNYLAILVKYV

180->190

NYLALLVKYV

182-191

YLALVIKFL

186-194

CGNVPIFKDGLGC GSCFEIKCDKPAE CSGKPVVVYIT

88-124

VLSVKVAALAGL IFSVLAASAAAK

$4-27$

LNPSIVFTYFTTFS LLTITCSCLHPKR $\mathrm{F}$

8-35

SGNPVTVVITDE

The

$45-56$
AKPVVGVDKYLL
KQGIVDVEYQRV
PCNY
69-96

YLAIKLLYQG

157-166

AGDLVKVSVK

186-195

\begin{tabular}{|c|c|c|}
\hline $\begin{array}{c}\text { CGSCFEIKCTK } \\
\text { PESCSGEPVL } \\
\text { VHIT } \\
92-116\end{array}$ & DGDVVAVDI & $170-175$ \\
\hline DGDVVAVDI & $\begin{array}{c}\text { CGSCFEIKCT } \\
\text { KPESCSGEPI } \\
\text { VVHIT }\end{array}$ & $\begin{array}{c}\text { IAAYHLDLS } \\
\text { GK }\end{array}$ \\
\hline 193-201 & 92-116 & $123-133$ \\
\hline DGDVVAVDI & $\begin{array}{c}\text { CGSCFEIKCT } \\
\text { KPESCSGEAV } \\
\text { TVT }\end{array}$ & $\begin{array}{c}\text { ELELQFRRV } \\
\text { KCKYP }\end{array}$ \\
\hline $193->201$ & $92->114$ & $152->165$ \\
\hline DGDVVAVDI & $\begin{array}{l}\text { CGSCFEIKCT } \\
\text { KPESC } \\
\text { SGEAVTVHIT }\end{array}$ & $\begin{array}{c}\text { ELELQFRRV } \\
\text { KCKYP }\end{array}$ \\
\hline 194-202 & 93-117 & 153-166 \\
\hline
\end{tabular}

KTVVDDVIPK

DGDVVGVDI

$\mathrm{K}$

CGSCFELKC TKPEACSG

247-256
PNYLALLVKY
V

197-206

DIVAVDI

96-112

KTVYDDVIP $\mathrm{T}$

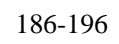

CGSCYQIRCS

201-207

YYPVAQYHF

DLSGT

123-136

SGKPVRVVIT DSCPGGPCLS

ESAHFDLSGT

GSSLVAPFSL
KLT

NAGVIQIQY

KRVECNYP

GVKLTFHV

DS

156-182

232-244

PGGPCVAES AHFDLSGT

NAGVLQIQY QKVKCNFP GAKVAFHV

DS

202-207

58-74

90-116

PNYLAIKLLY QG

TEVVGIDIAP

TDLVLSSR

112-123

CGACFQIRCK
DTTLCSR

126-139

56-63

TEVVAIDFAK

GHLAAAVSS

VG LYK

69-85 169-180

53-64
NSVLVGQSLS FRVT
SCGACFEIKC ANEPQWCHS GSPSIFIT

$215-228$ 80-106

174-183 


\section{Bioinformation}

\begin{tabular}{|c|c|c|c|c|c|c|c|}
\hline 1 & ACB & Sequence & SANAVALAALV & NYLAVLVEF & CGACYRIRCV & KTLVAKQVIP & YYPVAKYH \\
\hline \multirow[t]{3}{*}{6} & 45301 & & SVLLTYGCCAQ & & NNK & A & FDLSGT \\
\hline & .1 & & SPLNYTSLAKAS & & & & \\
\hline & & $\begin{array}{l}\text { Sequence } \\
\text { position }\end{array}$ & $5-38$ & 183-191 & $95-107$ & $248-258$ & $123-136$ \\
\hline 1 & ABJ & Sequence & NPPQHHFDLSQ & GCGSCYEIRCVN & AGDVHSVSV & YFNLVLITNV & MAGLLAML \\
\hline \multirow[t]{3}{*}{7} & 90221 & & PVFQHIAQYKA & DPKWCLPGTIAV & $\mathrm{K}$ & & VASAHAY \\
\hline & .1 & & $\begin{array}{c}\text { GVVPVSYRRVP } \\
\text { CR }\end{array}$ & TATNFC & & & \\
\hline & & $\begin{array}{l}\text { Sequence } \\
\text { position }\end{array}$ & $122->156$ & $78->107$ & $182->191$ & $170->179$ & $11->25$ \\
\hline 1 & AAT & Sequence & VGLSMACILSLR & YFNLVLIADVA & NTVLVGQALS & SGDIVKVSV & PMFLKIAEY \\
\hline \multirow[t]{2}{*}{8} & $\begin{array}{c}11859 \\
.2\end{array}$ & & CLMWM & & FRVR & $\mathrm{K}$ & $\begin{array}{c}\text { RAGIVPVSY } \\
\text { RRVPCR }\end{array}$ \\
\hline & & $\begin{array}{l}\text { Sequence } \\
\text { position }\end{array}$ & $4-20$ & $176-186$ & $217-230$ & $188-197$ & 139-162 \\
\hline 1 & BAC & Sequence & NPPQHHFDLSQ & GCGSCYEIRCVN & AGDVHSVSV & YFNLVLITNV & MAGLLAML \\
\hline \multirow[t]{3}{*}{9} & 67192 & & PVFQHIAQYKA & DPK & $\mathrm{K}$ & & VASAHAY \\
\hline & .1 & & $\begin{array}{c}\text { GVVPVSYRRVP } \\
\text { CM }\end{array}$ & $\begin{array}{c}\text { WCLPGSIVVTAT } \\
\text { NFC }\end{array}$ & & & \\
\hline & & $\begin{array}{l}\text { Sequence } \\
\text { position }\end{array}$ & $122-156$ & 78-107 & $182-191$ & $170-179$ & $11-25$ \\
\hline \multirow{3}{*}{$\begin{array}{l}2 \\
0\end{array}$} & BAC & Sequence & VSVSACLASLLI & YFNLVLVSNVA & NAVLVGQSLS & AGDIVRVSV & SCGACFEIK \\
\hline & 66787 & & SLMWV & & FRV & KGS & CA \\
\hline & & $\begin{array}{l}\text { Sequence } \\
\text { position }\end{array}$ & $4-20$ & $176-186$ & $217-229$ & 188-199 & $82-92$ \\
\hline
\end{tabular}

